\title{
Responsabilidad Social Corporativa (RSC) y Desarrollo Sostenible: una mirada desde la Declaración de Rio de 1992
}

William R. Avendaño C.*

Recibido:

Junio 23 de 2011

Aceptado:

Noviembre 30 de 2011

\section{Resumen}

La Responsabilidad Social Corporativa (RSC) es la contribución de las empresas a promover los cambios generados en las últimas décadas en materia de desarrollo sostenible en relación con el compromiso de los Estados de actuar responsablemente frente al medio ambiente. Esta tendencia ha tenido su auge a finales de los años 80 y comienzos de los 90 con la Declaración de Río en Brasil. Bajo esta perspectiva, se hace una revisión de lo que implica la Declaración de Río de 1992 para Colombia como Estado firmante y la RSC como un componente en las agendas de las organizaciones actuales. Para ello, se emprende una revisión exhaustiva de la literatura con la finalidad de realizar un acercamiento conceptual al término RSC. Se hace una revisión de la legislación en materia de RSC, analizando las posiciones de quienes están de acuerdo y los que se oponen. Se estudian las normas relacionadas con este ámbito en Colombia y se evalúa la necesidad de reglamentar en este país ejemplos llevados adelante por otros Estados.

Palabras clave: responsabilidad social corporativa, desarrollo sostenible, declaración de Rio

\section{Abstract}

The Corporate Social Responsibility is the contribution of the companies to promote the changes generated in the last decades on of sustainable development in relation with the commitment of the states to act responsibly towards the environment. This trend has had its heyday in the late 80's and early 90 with the declaration of Rio in Brazil. From this perspective, is a review of the implications the 1992 Rio Declaration to Colombia as a state party and the RSC as a component in the agendas of the actual organizations. For that, it make a thorough review of the literature in order to make a conceptual approach to the term RSC, the RSC legislation analyzing the positions of those who agree and those who oppose, the rules relationed to this area in Colombia and the need to regulate going to examples of other states.

Keywords: Corporate Social Responsibility, Sustainable Development, Rio Declaration.

\footnotetext{
*Docente - Investigador, Universidad Francisco de Paula Santander - Colombia. E-mail: wrac2008@hotmail.com
} 


\section{Introducción}

$\mathrm{L}$

a Responsabilidad Social Corporativa (RSC) es un tema de gran interés en la actualidad y constituye una respuesta a la demanda social, cada vez más grande, de propender por un desarrollo sostenible. Frente a ello, las organizaciones y compañías tienen la función de reconocer la capacidad que tienen de ocasionar serios y graves impactos negativos sobre el medio, y por lo tanto de la responsabilidad que subyace de la gestión de su actividad empresarial (Alea, 2007). Así, los avances sobre la RSC son el resultado de la dinámica de diversos factores que se imponen a partir de la globalización y la creación de conciencia de los diferentes actores sociales. Explica León (2009) que la RSC "se ha ido imponiendo en el escenario mundial como respuesta a (...) los cambios económicos, sociales y culturales" produciendo nuevas perspectivas en el rol del empresario, el Estado y la sociedad en general. En este orden, la RSC es el resultado de un llamado mundial y de compromiso de los países a contribuir con el Desarrollo Sostenible, aplicado a todas las organizaciones, ya que son actores activos claves en esta crisis y, por supuesto, pasivos en la medida que también se ven afectados (Raufflet, 2010).

La RSC es un tópico que ha sido estudiado y analizado por un gran número de autores, lo que ha conducido en los últimos años a que se convierta en un tema habitual en el ámbito de la gestión empresarial y ambiental (Moneva, 2006). Para Ariza, León y Gómez (2006) desde siempre ha sido una preocupación de meritorio y profundo análisis, la relación de los hombres con la naturaleza. Refiriéndose a ello explican que la "situación (...) ha sido discutida, estudiada y vivenciada desde los orígenes de la empresa comercial y a lo largo de su evolución y transformación, en sus etapas productiva, industrial, financiera, trasnacionalizada, globalizada y ahora financiarizada”.

Vidal (2010) estima, del mismo modo, que la RSC "está cada vez más presente en distintos ámbitos del mundo económico, social, político y académico (...) y se han llevado a cabo iniciativas públicas, empresariales, sindicales y sociales importantes para su extensión y la consolidación de su status político y social”.

Pese a todo el trabajo académico realizado para posicionarse como una tendencia, la RSC todavía no tiene un sustrato conceptual sólido, lo que da origen a muchos equívocos (Kirschner, 2004). De cierta manera no existe una unificación conceptual sobre el término de la RSC (Pinillos, 2005). Sin embargo, se logra percibir entre cada uno de estos aportes una relación con temas como la satisfacción de los clientes internos y externos, el desarrollo sostenible, la calidad, la preservación de los recursos, el impacto sobre la economía nacional, entre otros:

Desde la calidad hasta el mantenimiento y mejora del medio ambiente, pasando por la participación en el desarrollo económico y social de los países en los que opera, por poner algunos ejemplos, forman parte ya de lo que la gente, la opinión pública, espera de una empresa. Es decir, son parte ya de su responsabilidad (García-Marzá, 2004).

Para la Asociación Española de Contabilidad y Administración de Empresas (AECA) (2004), la RSC puede definirse como el compromiso voluntario de las empresas con el desarrollo de la sociedad y la preservación del medio ambiente, desde su composición social y un comportamiento responsable hacia las personas y grupos sociales con los que interactúa. Desde la perspectiva de la AECA, la RSC es un compromiso voluntario lo que resulta de cierto modo cuestionable, si se atiende al marco normativo internacional e interno de los países que de una u otra manera exigen responsabilidad en las actuaciones de las empresas y organizaciones.

Las empresas actuales son organizaciones generadoras de beneficios no sólo económicos sino también sociales (Vargas y Vaca, 2005), y desde esa perspectiva están llamadas 
a implementar acciones socialmente responsables en cada una de las etapas del proceso productivo en función de mitigar los efectos nocivos sobre la sociedad en general y el medio ambiente. Pero en contravía de esto, se ha olvidado que una empresa es una asociación de agentes económicos que incluye otros individuos diferentes a los que están relacionados directamente con ella (Rodríguez, 2003).

Las organizaciones tienen un papel fundamental de tipo económico, ambiental y social del cual deben ser conscientes las mismas aun más cuando el desarrollo sostenible es una urgencia en las sociedades modernas (Vélez, 2010). De este precepto surge la necesidad de reformas urgentes y pertinentes, las cuales han sido señaladas desde las últimas décadas y con especial fuerza en los últimos 20 años por organizaciones mundiales, gobiernos y expertos. Todo ello ha tenido un cierto impacto en el mundo. En América Latina, por ejemplo, en un estudio realizado por Vives, Corral e Isasi (2005) encontró que dentro de las Micro, Pequeñas y Medianas Empresas (MiPyMEs), las cuales representan el $90 \%$ de la región, un 14,5\% implementan en alto grado la RSC, un 34\% tienen una aplicación media y un $47,9 \%$ baja. Sólo el 3.5\% de las MiPyMEs no implementan ningún tipo de actividad de RSE.

De acuerdo con éste primer acercamiento, el objetivo del artículo es hacer una revisión de la RSC desde el ámbito legal en Colombia, el cual incluye un acercamiento conceptual a las iniciativas mundiales en materia de Desarrollo Sostenible, a la reglamentación interna en Colombia y a determinados avances manifiestos en virtud del cumplimiento de este marco jurídico.

\section{Materiales y métodos}

El estudio es de tipo descriptivo toda vez que el objeto del mismo es identificar las características de ciertos fenómenos, en este caso, los relacionados con la RSC y las normas jurídicas que giran alrededor de ella. Como señala Sabino (1992), la principal preocupación de la investigación descriptiva es la de "describir algunas características fundamentales de conjuntos homogéneos de fenómenos". Para lograr el objetivo propuesto, se recurrió al análisis de las iniciativas mundiales sobre Desarrollo Sostenible, con las cuales se ha comprometido el Estado colombiano; en el mismo curso se ejecuta la revisión de la literatura acerca de la RSC para extraer sus principales elementos y a su vez una exploración del marco legal interno.

\section{Resultados y discusión}

\section{El compromiso del estado colombiano con el desarrollo sostenible}

El desarrollo sostenible es una premisa que tiene su origen en la década de los ` 80 con el reporte titulado "Nuestro Futuro Común de la Comisión Mundial de Medioambiente y Desarrollo en 1987" y que se traduce en la facultad de la humanidad para garantizar la satisfacción de las necesidades actuales sin arriesgar la capacidad de satisfacer sus propias obligaciones a las generaciones futuras.

A partir de esta publicación se ha convocado a los Estados y sus gobiernos para que estos atiendan la grave situación que representa la degradación del medio ambiente, el cambio climático, la deforestación de los bosques, la emisión indiscriminada de gases, entre otras amenazas. El compromiso real de los gobiernos se materializa con la Cumbre de la Tierra y la Conferencia Mundial para el Desarrollo Sostenible de Naciones Unidas (World Summit on Sustainable development) en el 2002.

En el año de 1992 en Río de Janeiro, Brasil, se llevó a cabo la Cumbre de la Tierra o Conferencia de las Naciones Unidas sobre el Medio Ambiente y el Desarrollo (CNUMAD), en la que 172 gobiernos aprobaron tres 


\section{Responsabilidad Social Corporativa (RSC) y Desarrollo Sostenible: una mirada desde} la Declaración de Rio de 1992 acuerdos en materia de Desarrollo Sostenible ${ }^{1}$. La declaración, uno de los tres acuerdos, adoptada por los gobiernos está constituida por 27 principios entre los que se destacan: el derecho de las personas a una vida saludable (1), el derecho de los Estados a aprovechar sus recursos y el deber de velar por las actividades que se realizan en su jurisdicción (2), equivalencia entre desarrollo y necesidades sin arriesgar las generaciones presente $\mathrm{y}$ futuras (3), integración de la protección del medio ambiente en la agenda de desarrollo sostenible (4), la cooperación y solidaridad mundial en función de conservar, proteger y restablecer la salud e integridad del ecosistema de la Tierra (7), la reducción y eliminación de modalidades de producción y consumo insostenibles (8) a través del aumento del saber científico y el uso de tecnologías (9), la vinculación de todos los ciudadanos de forma participativa y el desarrollo de estrategias para la transmisión de información, materiales y actividades por parte de las autoridades públicas (10), la promulgación de leyes eficaces para la protección del medio ambiente (11), la promoción de un sistema económico pertinente con el desarrollo sostenible (12) y el emprendimiento de la evaluación del impacto ambiental (17).

Con esta Declaración, se evidencia el compromiso adquirido por el gobierno colombiano en el desarrollo de actividades que garanticen de forma tangible la protección del medio ambiente y el desarrollo sostenible. Por lo tanto, después de casi 20 años de la fecha en que se suscribe la Declaración de la Tierra, se esperaría ver el resultado claro y materializado de los avances significativos que habrá desarrollado el Estado en este campo.

Por lo tanto y teniendo en cuenta la mención a los avances que se han ejecutado en torno al desarrollo sostenible tal cual se plantea en la introducción, se destaca la actuación de las empresas como actores sociales primordiales que encuentran en la RSC, la traducción de la
Declaración de Río, una guía para mantener la tendencia de las organizaciones de actuar responsable y consecuentemente con los acuerdos ya mencionados.

\section{La Responsabilidad Social Corporativa (RSC)}

La RSC hoy por hoy se está convirtiendo en una "licencia social para que puedan operar las empresas en el mundo y en Colombia" (McPhail, K., 2000), y ha permitido redefinir el rol de todos los actores sociales y es por este motivo que se considera también que "despierta un gran interés por parte del sector empresarial, las escuelas de negocio, los inversores e incluso algunos gobiernos" (De la Cuesta, 2005).

La RSC la asume, de una manera amplia Lindbaek (2003) quien explica que "la responsabilidad social corporativa (...) es la responsabilidad que la corporación o la compañía tiene o asume frente a la sociedad en general". Pero como se ha mencionado, se está frente a un constructo que ha sido estudiado y definido de varias maneras. Por ejemplo, para Encinas (2000), la RSC es "una serie de conductas exigibles a las empresas por parte de esos interlocutores en función de la actividad que realiza, cuyo adecuado cumplimiento determinará que ésta sea aceptada o rechazada en sus diferentes aspectos (social económico, medioambiental)".

Sin embargo, no puede confundirse la RSC con la filantropía corporativa, entendiéndose la primera como la responsabilidad de los gerentes, dueños de empresas, accionistas, desde el ámbito económico, laboral, tributario, ambiental y de una manera más significativa lo social (Sanborm y Portocarrero 2003). Mientras que la filantropía corporativa no es una responsabilidad exigible a las empresas por lo que depende sólo de los actos altruistas y solidarios de sus directivos. 
Por su parte, Schmidheiny (2004) define la RSC como "el compromiso de las empresas de contribuir al desarrollo económico sostenible trabajando con los empleados, sus familias, la comunidad local y la sociedad en general para mejorar su calidad de vida”. Cabe acotar que se trata de un compromiso permanente y exigible y no de actos de filantropía corporativa como se hace mención en el párrafo anterior.

La literatura que versa sobre este asunto es muy diversa como resultado de las distintas perspectivas de la cual se ha nutrido, por ejemplo académica, económica, empresarial, entre otras. Así que los aportes teóricos pueden tomar impredecibles matices. Garriga y Melé (2004) citado por De la Cuesta (2005) describe que existen cuatro grupos de teorías dentro de las que se incorpora la RSC: instrumentales, políticas, integrativas y éticas.

La comprensión instrumental de la RSC es la entendida como aquella que genera valor a la empresa; la visión política es la que entiende la RSC como parte del poder de las organizaciones; la perspectiva integrativa de la RSC promueve la satisfacción de las necesidades de los "stakeholders" (Friedman, 1962); y la mirada ética de la RSC es la que permite abordarla desde el ámbito ético respecto de la sociedad. Así, en un intento por agrupar estas nociones, es permisible asumir la RSC como el conjunto de acciones de la organización coordinadas con el direccionamiento estratégico, los planteamientos éticos, las necesidades de la comunidad con la que tiene relación directa e indirectamente y los postulados normativos de tipo jurídico.

Otro abordaje expuesto, Aceituno (2009), considera que las causas de la tendencia de la RSC obedecen a: a) la nueva cultura del ciudadano, b) la mayor vertebración de la sociedad civil mediante la creación de diferentes grupos sociales, c) el nacimiento de una nueva sensibilidad social como producto del nuevo marco y, d) los impactos de diversas organizaciones del entorno.
Visto entonces desde una gran panorámica se entiende que los anteriores planteamientos requieren de una nueva acción social y de una mayor aceptación por parte de las empresas de asumir una responsabilidad frente a la sociedad, la cual debe estar basada no solo en la cooperación y en lograr contribuir en el equilibrio social (cohesión y conexión entre los grupos sociales y la ciudadanía), sino también hacer gran énfasis en la protección del medio ambiente, en el desarrollo económicosocial y en la creación de riqueza y empleo. Como explica Salamon (1999), al referirse a la responsabilidad de las empresas no lucrativas, pero de igual forma desde el marco social, es necesario forjar un modo distinto de formación gerencial (...) que tenga en cuenta los valores y el espíritu característicos del sector [y] quedan por dar pasos importantes para fomentar la capacidad de formación". En esta misma línea advierte Salamon (1999) de una manera totalmente válida y aplicable a las organizaciones desde la perspectiva de la RSC que hay una "necesidad de una nueva cultura cívica, que la propia globalización requiere, va a exigir el concierto y el compromiso conjunto (...) capaz de posibilitar la creación de verdaderas oportunidades y de nuevas opciones para todos".

Las empresas, como forma particular de una organización (Berbel, Reyes y Gómez, 2007), están llamadas a abordar cientos de problemas sociales, pero sólo algunas son oportunidades de hacer una real diferencia para la sociedad o de conferir una ventaja competitiva, teniendo como función la de satisfacer necesidades, pero también procurar el desarrollo pleno de su potencial, (Velásquez, 2000). Es así que si las organizaciones hacen las elecciones correctas y crean iniciativas sociales enfocadas, proactivas e integradas, en concierto con sus estrategias centrales, se distanciarán cada vez más del resto (Porter y Kramer 2006). Se trata de una oportunidad para aumentar la competitividad de las empresas y esto se logra, según Porter y Kramer (2006), al vincular articuladamente la responsabilidad social en cada una de las fases de la cadena de valor, en otras palabras, cuando se hace aplicación del 
"principio de valor compartido que implica la creación de valor económico de una manera que también crea valor para la sociedad para que ésta haga frente a sus necesidades y desafíos" (Porter y Kramer, 2011).

Cabe mencionar que el concepto de valor compartido se encuentra en un primer estado, es decir, en plena génesis y sólo hasta ahora toma fuerza cuando la economía capitalista, con sus crisis, ha puesto en serios problemas a un sin número de empresas en el mundo. Además de ello, se suman conductas irresponsables de grandes organizaciones como la Britsh Petroleum cuyo derrame de petróleo sobre el Golfo de México resultó ser un desastre ecológico y una amenaza aun no calculada; o Trafigura, compañía de transporte británica, quien pagó para deshacerse de 400 toneladas de gasolina de baja calidad y que fueron esparcidas en cercanías a la ciudad de Abidjan en el 2006.

Malagón (2010) también analiza el binomio RSC y valor agregado. En su obra aborda esta relación y explica que la RSC y la generación de valor (uno de los objetivos centrales de las organizaciones) "no se oponen con la búsqueda del bien común". También describe la manera como "en el marco de la sociedad de la información y el conocimiento", hablar de generación de valor es también estimar "la perspectiva del Capital Humano, del Capital Social, y del Capital Moral" pues estas son, en sí, "fuentes poderosas de valor y de riqueza en el mundo de hoy".

Las brechas entre los países desarrollados y los que se encuentran en desarrollo son cada vez más grandes. Y resulta la RSC, como lo señalara Porter y Kramer (2006), una oportunidad para los países en desarrollo. Amplía las justificaciones Visser (2008) sobre esta posición al explicar que la RSE beneficia a los países en desarrollo porque:

a. los países en desarrollo representan la mayoría de las economías en rápida expansión, y por lo tanto, con mercados lucrativos en crecimiento para los negocios;

b. los países en desarrollo, donde se dan crisis sociales y ambientales, por lo general, revisten especial gravedad;

c. en los países en desarrollo la globalización, el crecimiento económico, la inversión, y la actividad empresarial, es probable, que tengan impactos sociales y ambientales más dramáticos (tanto positivos como negativos); y

d. los países en desarrollo presentan un conjunto distintivo de la RSE (en sus desafíos y agendas) muy diferentes a los que se enfrentan en los países desarrollados mundialmente.

Es advertido como el contexto propio de los países en desarrollo, como Colombia, donde se presentan mayores razones para implementar prácticas de RSC. Suscita la duda, en torno a esto, la necesidad o no de incorporar la RSC dentro de los Estados como un acto meramente voluntario o una responsabilidad legal taxativa. Otro elemento que se agrega al complejo panorama es el de la responsabilidad laboral de las empresas, pues se ha comprobado que las políticas de subcontratación de mano de obra conducen a la violación de los Derechos Humanos en muchos de los casos. Así lo explica Prieto-Carrion (2006) quien analiza dicha problemática en su investigación titulada Corporate Social Responsibility in Latin America: Chiquita, Women Banana Workers and Structural inequalities.

Pinillos (2005) asume la RSC como una tendencia de tipo filantrópica, como la financiación de programas sociales o programas de voluntariado cooperativo para sus trabajadores. En el caso que aquí se plantea se asume que a la RSC como la obligación que implica para las empresas beneficiarse del entorno natural y social, y las consecuencias de esto en relación con la premisa de asegurar la satisfacción de necesidades de las generaciones futuras. Por ello, la legislación no buscaría regular la excelencia sino el cumplimiento de un mínimo de requerimientos imprescindibles para contribuir al desarrollo sostenible. 
En diferentes partes del mundo se ha podido determinar que la implementación de la RSC en las empresas ha evidenciado excelentes resultados tanto sociales como medioambientales, los siguientes autores establecen cuales son dichos beneficios: aumentan los ingresos organizacionales y el valor económico de estas (Simpson y Kohers, 2002; McWilliams y Siegel, 2000; Griffin y Mahon, 1997; Aupperle, Carroll y Hatfield,1985); disminuyen el riesgo (Moore, 2001; Orlitzky y Benjamin, 2001); atraen o retienen empleados (Backhaus et al. 2002; Turban y Greening, 1997); incrementan la lealtad y mejoran la imagen o reputación corporativa (Maignan etal, 1999; Brown y Dacin, 1997). De igual manera, la ventaja competitiva que genera para las empresas el desarrollar una estrategia basada en lo ambiental, logra tal y como lo describen (Trujillo y Vélez, 2006), una reducción de costos en sus procesos, un mejor aprovechamiento de los recursos, agrega valor al producto y lo posiciona en el mercado atendiendo a la consciencia social de quienes lo consumen.

Finalmente cabe destacar que la RSC es un aporte de las organizaciones en el desarrollo sostenible de los pueblos, y por ello, en virtud del marco jurídico internacional, debe ser reglamentado por el Estado y de esta manera, pasar de una posición que asume la RSC como discrecional de las corporaciones a constituirse en una obligación de carácter legal que exige de estas últimas la inclusión de medidas exigibles y coercitivas necesarias. En contraposición a este argumento, Pinillos (2005), al intentar responder a la pregunta idebe existir una norma legal que regule la RSC?, refiriéndose al caso de las empresas en España, menciona que "legislar sobre la excelencia, es decir, la gestión excelente de los procesos en relación de una empresa con sus stakeholders no parece ser posible. Por otro lado, legislar la forma y el fondo de la filantropía empresarial, pues tampoco tiene mucha lógica".

A simple vista, dicha afirmación parecería tener validez sin embargo, no se puede interpretar su explicación fuera del contexto en el que surgió.

\section{El estado colombiano y la materialización de la RSC en el territorio}

La responsabilidad empresarial en relación con el desarrollo sostenible en el país, es un tema mas ético y cultural que legal. Sin embargo en una sociedad en la que el peso moral de las acciones es casi irrelevante (Bernal, 2009), debe tomarse entonces el marco jurídico, que en nuestro país aparece casi nulo, al igual que el desarrollo de políticas y acciones públicas en lo que corresponde a la RSC. En la Constitución Política de 1991 se establecen algunos lineamientos que son el producto de una visión del deber ser de la sociedad colombiana. De esta manera, el Capitulo III De los Derechos Colectivos y del Ambiente, del Título II De los derechos, las garantías y los deberes, el cual menciona el derecho a un ambiente sano (art. 79) y la potestad de la ley para regular la producción de bienes y servicios (art. 78). De manera directa, y en concordancia con la Declaración de Río la Carta Política menciona que:

"El Estado planificará el manejo y aprovechamiento de los recursos naturales, para garantizar su desarrollo sostenible, su conservación, restauración o sustitución. Además, deberá prevenir y controlar los factores de deterioro ambiental, imponer las sanciones legales y exigir la reparación de los daños causados. Así mismo, cooperará con otras naciones en la protección de los ecosistemas situados en las zonas fronterizas".

En el 2007, se intentó mediante el Proyecto de Ley No 31, definir normas sobre la Responsabilidad Social y medioambiental de las empresas. Este proyecto de ley tomaba como ámbito de aplicación todas las medianas y grandes empresas y, opcionalmente, las micro y pequeñas empresas, las cuales obtendrían incentivos en caso de acogerse a esta normatividad. 


\section{Responsabilidad Social Corporativa (RSC) y Desarrollo Sostenible: una mirada desde la Declaración de Rio de 1992}

Visto como un avance el artículo $5^{\circ}$ obligaba a las empresas a realizar un informe cada año en el mes de diciembre y su respectiva publicación con la información relacionada sobre el impacto ambiental, social, económico y financiero de su actividad, además de las políticas y programas relacionados con la RSC implementadas.

Sumado a lo ya expuesto, el proyecto de ley proponía la constitución de un Comité (Consejo de Responsabilidad Social Empresarial CRSE) encargado de expedir normas y evaluar el estado de la responsabilidad empresarial y medio ambiental en Colombia.

De acuerdo con la exposición de motivos, la RSC seria observada desde tres aspectos fundamentales:

- Laboral: cumplimiento de las normas de trabajo, respeto al trabajador en todos los niveles jerárquicos y puesta en marcha de códigos de conducta y principios de la Organización Internacional del Trabajo (OIT).

- Económico: elaboración de cuentas transparentes y públicas, e inversiones socialmente responsables (utilizando criterios éticos y de exclusión, a la hora de invertir).

- Ambiental: respetando el medioambiente y usando sellos de calidad ecológica, o información directa al consumidor de cómo se obtuvo el producto, o se prestó el servicio.

Sin embargo y en detrimento de las ventajas que de este podrían resultar, el proyecto de ley no fue aprobado con base a la ponencia para primer debate, en donde se expuso que el mismo proyecto desvirtuaba la filosofía de la RSC, por cuanto establecía la obligación de rendir informes anuales por parte de las empresas en materia de RSC. Frente a ello, se debe destacar que la misma Declaración de Río exige de los Estados la implementación de acciones para diagnosticar el nivel de desarrollo sostenible y conforme a ello solicita la publicación de la información para la toma de decisiones pertinentes. De tal forma se corrobora que la intención de los autores de la iniciativa, no era otra que dar cumplimiento a aquellos compromisos que ha adquirido el Estado colombiano y conseguir un avance en dicho objetivo.

En consecuencia de lo mencionado anteriormente queda evidenciado que Colombia no se encuentra en un nivel tan avanzado como Estados Unidos u otros países europeos, adicionalmente se desconoce una de las tendencias que se lleva a cabo en materia legislativa internacional:

El derecho comparado (...) ha incluido en (el) sistema legislativo dos piezas concretas. La primera de ellas es el reporte de sostenibilidad en un sentido amplio, en el que se establece la obligatoriedad de las empresas cotizadas a realizar informes sobre esta materia (Pinillos, 2005).

Un ejemplo de ello es Francia, que divide el informe de RSC en cuatro ámbitos: comunidad, recursos humanos, estándares laborales y gestión del medio ambiente.

Regresando al Estado colombiano, y a la negativa de aprobar el proyecto de ley, se encuentra que la ponencia ante la Cámara de Representantes del Congreso de la República, también acude a estimar la RSC como un acto filantrópico, un conjunto de acciones que no tienen carácter vinculante sino una estrategia de competitividad. Por lo que afirman que obligar a la empresa a desarrollar programas de RCS desvirtúa la esencia de la misma.

Se encuentra también que el Departamento Administrativo Nacional de Estadística, DANE, en su base de datos detenta una publicación de desarrollo sostenible en donde se hace un diagnostico de la situación en Colombia en este campo. Esta corresponde al año 2007, siendo Presidente de la República el Dr. Álvaro Uribe Vélez, y resultan ser los primeros pasos de la Etapa I del proyecto destinado a "elaborar una herramienta de consulta y difusión de variables económicas, sociales, demográficas y ambientales que 
sirvan para la formulación de políticas y toma de decisiones, bajo el enfoque socio ecológico propuesto por la CEPAL". Dicha herramienta hubiese resultado efectiva para alcanzar el objetivo de coaccionar el actuar de las organizaciones.

Finalmente y haciendo un abordaje respecto de algunos alcances positivos que se han tenido en materia de legislación se encuentra el Decreto 1299 de 2008. Esta herramienta jurídica ha tenido por objeto el mejoramiento de las actividades ambientales de las empresas por cuanto exige el cumplimiento de la normatividad ambiental vigente. A diferencia de los enunciados que se proponen en la Declaración de Rio de 1992, en este Decreto se destaca la obligatoriedad tal cual lo manifiesta el segundo inciso del artículo 8. : "El incumplimiento de las obligaciones contenidas en el presente decreto dará lugar a las sanciones respectivas, según el caso" por lo tanto la discrecionalidad de la gestión empresarial ambiental no es optativa sino impositiva so pena de hallarse sujeto de una sanción es caso de incumplimiento. $Y$ en el mismo cause se fija entonces una actividad de vigilancia y control por parte de las autoridades ambientales.

Siendo este decreto un notable avance en la regulación de la actividad ambiental por parte de las Organizaciones, su carácter vinculante está centrado en el manejo de la gestión industrial dejando nuevamente un vacío en los demás devenires que afectan a la sociedad por parte de las empresas.

Haciendo mención sobre otros alcances positivos se encuentra que en Colombia en varias oportunidades se ha regulado sobre las actividades consideradas como de alto riesgo y que por su misma naturaleza tienden a desequilibrar el ambiente, tal cual es el caso de la gestión de residuos peligrosos. Un ejemplo de ello se encuentra en las normas dictadas desde la expedición del Código de Recursos Naturales Renovables (CRNR) decreto ley 2811 de 1974, el Código Sanitario Nacional (CSN) Ley 9 de1979, la Ley 99 de 1993, la Ley
142 de 1994, la Ley 430 de 1998 y la Resolución 2309 de 1986. Expedida por el Ministerio de Salud modificada por el Decreto 4741 de 2005 del Ministerio de Ambiente y Desarrollo Territorial. Dicha normatividad ha dado luces sobre el manejo adecuado que deben tener las empresas sobre temas puntuales y específicos en materia ambiental, y del que son objeto de reglamentación, regulación y sanciones frente al desacato de la norma.

En Colombia el despliegue jurídico en materia ambiental no ha sido exclusivo de las actividades de alto riesgo y su paulatina regulación, se puede destacar también el desarrollo del que ha sido sujeto el tema de las licencias ambientales, se evidencia una favorable panorámica en el tema, el Gobierno Nacional expidió una nueva reglamentación al Título VIII de la ley 99/93 (ley del medio ambiente) en lo relativo a las licencias ambientales. Éstas son las autorizaciones que otorgan las autoridades ambientales competentes (Minambiente, entidades territoriales, entre otras) para la ejecución de un proyecto, obra o actividad, que pueda producir deterioro grave a los recursos naturales renovables o al medio ambiente o introducir modificaciones considerables o notorias al paisaje. Dicho lo anterior se puede destacar entonces que bien el intento de legislar sobre el tema ha presentado grandes avances pero también grandes retrocesos.

En este orden de ideas y siguiendo con el lineamiento de presentar las actuaciones que versan sobre la RSC, no se puede obviar los mecanismos de participación y de control que se encuentran establecidos en la legislación colombiana. La Ley 99/93 en su artículo 72 establece la audiencia pública siendo esta un mecanismo de participación que le ofrece a la comunidad, a las autoridades, a las organizaciones y a la autoridad ambiental, la posibilidad de conocer, informarse e intercambiar criterios sobre la conveniencia de una obra o actividad que se desarrolle o pretenda desarrollarse y que pueda causar impacto al ambiente o a los recursos naturales renovables. Dichas Audiencias 
pueden ser solicitadas por el Procurador General de la Nación o el Delegado para Asuntos Ambientales; el Defensor del Pueblo; el Ministerio del Medio Ambiente; las demás autoridades ambientales como las Corporaciones Autónomas Ambiental CARs; los Gobernadores; los Alcaldes.

Fijándose entonces la disyuntiva entre la exigencia y la permisibilidad con la que el Estado Colombiano trata el tema de la RSC se evidencia una vez más que si bien regula las actividades realizadas por los particulares, resulta de apremiante urgencia la constitución de estrategias y políticas públicas que fortalezcan el liderazgo y la autoridad social de las organizaciones que son poderosas, a fin de reglamentar los temas concernientes al medio ambiente y el desarrollo sostenible (Carvajalino 2002; Paoli 2002) ya que los líderes empresariales son agentes morales que ostentan una enorme capacidad de coerción moral con los otros miembros de la sociedad (Solarte, s.f.).

En otras palabras tal como lo define Rojas (2002): "el estado colombiano es débil y más bien necesita legitimarse". Además existen en nuestro país grandes incertidumbres con respecto al tema y las múltiples maneras en que puede ser interpretado, aplicado y evaluado, que no permite que este instrumento sea utilizado correctamente, (Heincke, 2005). Por lo tanto, las organizaciones modernas no sólo deben preocuparse por reportar utilidades sino también por llegar a desarrollar acciones que ayuden a contribuir con una mejora de la sociedad y preservar el medio ambiente (Rodríguez, 2010).

Visto de esta forma, el sector privado como los demás sectores de la sociedad civil, deben comprometerse, no sólo con compartir los espacios de discusión y acción social, sino también deben asumir responsabilidad legal y moral, sin descuidar las responsabilidades como causantes y receptores de impactos negativos y positivos (Ramírez y Segura, 2010).
Deshilvanado el tema de la RSC, las acciones que se han realizado y las negativas que aún se presentan en el actuar colectivo de las organizaciones y la carencia de exigibilidad estatal, podemos afirmar, que aún falta un largo camino en materia legislativa para recrear lo que representa la RSC y el cumplimiento de la Declaración de Río y demás instrumentos aprobados por el gobierno colombiano. Se evidencia una clara desarticulación entre las normas internacionales, con las normas internas y con las acciones de las empresas y entidades encargadas de proporcionar la información necesaria para generar cambios positivos.

\section{Conclusiones}

Se observó que a partir de la Declaración de Río en 1992 se inició un cambio en la comprensión del medio en el que habitamos. De ello, se desprendió el compromiso de los países del mundo por llevar a cabo acciones eficaces para promocionar el desarrollo sostenible dentro de sus jurisdicciones.

Paralelamente a esta nueva actitud de los países, se gestaba en el mundo una visión diferente de las empresas y de su papel en la sociedad, creándose la perspectiva RSC que implica el impacto positivo de las organizaciones con sus stakeholders y con el medio ambiente. Sin embargo, poner en marcha programas de este tipo implica investigación, inversión y bastante trabajo. De allí, que no todas las empresas encuentren la RSC como una oportunidad a menos que generen utilidades (perspectiva instrumental).

De este panorama, surge la necesidad de reglamentar la RSC en lo que tiene relación con el desarrollo sostenible, con el objetivo de garantizar un mínimo de requerimientos para las empresas y que permitan el éxito del desarrollo sostenible. Muchos países han incorporado marcos jurídicos internos con un gran impacto positivo (Pinillos, 2005). 
Para el caso de Colombia, la situación resulta desfavorable frente a otros países porque no hay un marco jurídico efectivo y hasta ahora se está iniciando la etapa de diagnostico que busca encontrar la características del país en estos aspectos. Para Colombia, como país en desarrollo, la RSC resulta todo un desafío en el marco de los objetivos de reducir la pobreza, el hambre y la enfermedad; aumentar las posibilidades de supervivencia de madres y niños recién nacidos, mejorar el nivel de educación, alcanzar una sociedad caracterizada por la igualdad de oportunidades y el goce de un ambiente sano (Visser, 2008) ¿Cuál es el papel de las empresas en abordar los temas críticos del desarrollo humano y la sostenibilidad ambiental en los países en desarrollo?

No es prudente finalizar sin antes exponer un tema relevante y relacionado con la RSC. En el desarrollo de las sociedades y el crecimiento de la economía, la RSC juega un rol importante. En la medida que dicha conciencia se incorpore dentro de la cultura, la transformación será menos o más evidente en el estado de bienestar de las personas. Y en éste orden, no será posible una modificación de éste tipo, sin una inversión suficiente en la educación. Por ello, la educación afecta no sólo el crecimiento económico sino además el efecto de la inversión total de la economía sobre el crecimiento (Malagón, 2010).

\section{Agradecimientos}

El autor agradece a la Universidad Francisco de Paula Santander y a Alberto Díaz Montes quien hizo aportes importantes para la construcción de este trabajo.

\section{Referencias bibliográficas}

Aceituno, Pedro (2009); La responsabilidad social corporativa como instrumento de atracción del conocimiento científico para superar la crisis económica actual. Facultad de Administración y Dirección de Empresas Universidad a Distancia de Madrid. Artículo consultado el 12 de mayo de 2011 en:

http://www.aeca.es/pub/on_line/ comunicaciones_xvcongresoaeca/cd/8h.pdf

Alea García, Alina (2007); Responsabilidad Social Empresarial. Su contribución al desarrollo sostenible. En Revista Futuros, Vol. V, No 17. ISBN 84-7299-637-9. Articulo consultado el 17 de mayo de 2011 en:

http://www.revistafuturos.info/raw_text/raw_ futuro17/resp_social_empr.pdf

Ariza Buenaventura, Danilo, León Paime, Fredy y Gómez Villegas, Mauricio (2006); Una aproximación a los perfiles ambientales de la empresa. En Revista Innovar, Vol. 16, No 028. p.p. 57-74. ISSN 01215051

Asociación Española de Contabilidad y Administración de Empresas (AECA) (2004); Marco conceptual de la responsabilidad social corporativa. Madrid: AECA.

Aupperle, K.E., Carroll, A.B. y Hatfield, J.D. (1985); An empirical examination of the relationship between corporate social responsibility and profitability. En Academy of Management Journal, Vol. 28, No 2. p.p. 446-463.

Backhaus, K.B.; Stone, B. A. y Heiner, K. (2002); Exploring the relationship between corporate social performance and employer attractiveness. En Business and Society. Vol. 41, No. 3. p.p. 292-318. ISSN 0122-820X 
normas de gestión e información. En Revista Innovar, Vol. 17, No 029. p.p. 27-47. ISSN 01215051

Bernal, Andrea Yolima (2009); Responsabilidad ambiental de las empresas: un compromiso ético con el desarrollo sostenible. En Management, Vol. XVIII, No 31. p.p 91 103. ISSN 0122-6681.

Carvajalino, Guillermo (2002); Philanthropy and Education in Colombia. Comentarios presentados en el taller sobre Philanthropy and Social Change in the Americas: Strategies and Lessons. En Harvard University PASCA, May 22-24.

Prieto-Carrion, M. (2006); Corporate Social Responsibility in Latin America: Chiquita, Women Banana Workers and Structural inequalities. Bristol, Spring, p.p. 2-10.

Comisión Mundial de Medioambiente y Desarrollo (1987); Nuestro Futuro Común.

Constitución Política de Colombia de 1991.

Declaración de Río sobre el Medio Ambiente y el Desarrollo, 14 de junio de 1992, Río de Janeiro, Brasil.

De la Cuesta González, Marta (2004); Inversiones socialmente responsables. Salamanca: Universidad Pontificia de 56 Salamanca.

Departamento Administrativo Nacional de Estadísticas DANE (2007); Iniciativa latinoamericana y caribeña para el desarrollo sostenible: Indicadores de Seguimiento. Primera edición, Bogotá: DANE.

García - Marzá, Domingo (2004); La responsabilidad social de la empresa: una definición desde la ética empresarial.
Universitat Jaume I. En: R.V.E.H. № 12 - III.

Encinas Duval, Beatriz (s.f.); Marco conceptual de la responsabilidad social de las empresas. La dimensión social de la empresa. En X Jornadas de investigadores en economía social y cooperativa: Emprendedores, economía social, democracia económica. CEPES Andalucía, CIRIEC España, Universidad de Jaén. Artículo consultado el 16 de mayo de 2011:

http://www.catedracajamarupm.es/ Catedra_CajamarUPM/Ponencias_y_ Comunicaciones_files/7.pdf

Friedman, M., (1962); Capitalism and freedom. Chicago: University Press.

Griffin, J.J. y Mahon, J. F. (1997); The corporate social performance and corporate financial performance debate: twenty-five years of incomparable research. En Business and Society, Vol. 36, No 1. p.p. 5-31

Heincke, Monica (2005); La Responsabilidad Social Empresarial: iUna herramienta para el desarrollo local sostenible en Colombia? Revista Opera, Volumen 5, No 005. p.p. 5574. ISSN 1657-8651.

Kirschner, Ana María (2004); La Responsabilidad Social de la Empresa. En: Revista Nueva Sociedad, № 202, Marzo-Abril 2006. Artículo consultado el 18 de mayo de 2011 en:

//www.nuso.org/upload/articulos/3343_1.pdf

León López, Guillén (2009); La Responsabilidad Social de las Empresas carboníferas en la ciudad de Santa Marta. Ponencia IX Congreso Latinoamericano ALENE Universidad Sergio Arboleda. Bogotá, Colombia - septiembre 24 y 25. 
Lindbaek, Jannik (2003); La ética y la Responsabilidad Social Empresarial. Seminario Internacional "Capital Social, Ética y Desarrollo: Los desafíos de la gobernabilidad democrática" Caracas, 26 de junio del 2003. Artículo consultado el 17 de mayo de 2011 en:

http://www.insumisos.com/lecturasinsumisas/ $\mathrm{L}$ a $\% 20$ e ti c a $\% 20$ y 201 a $\% 20$ responsabilidad\%20social.pdf

Maignan, I., Ferrell, O.C. y Hult, G. T. M. (1999): Corporate Citizenship: cultural antecedents and business benefits. En Journal of the Academy of Marketing Science, Vol. 27, No. 4. p.p. 455-469.

Malagón, Víctor Hugo (2010); Ética y responsabilidad: el nuevo reto de generación de valor en las organizaciones. Bogotá: Corporación Siglo XXI \& KAS.

McPhail, K., (2000); How oil, gas and mining projects can contribute to development. En Finance and Development, Vol. 37, No 4. p.p. 46-50.

McPhail, K. a. D., Aidan (1998); Integrating Social Concerns into Private Sector Decisionmaking. A Review of Corporate Practices in the Mining, Oil and Gas Sectors. En World Bank Discussion Papers, No. 384.

Mc Williams, A. y Siegel, D. (2000); Corporate social responsibility and financial performance: correlation or misspecification. En Strategic Management Journal.

Melé, Domènec; Garriga, Elisabet y Guillén, Manuel (2002); Políticas de ética empresarial en las 500 mayores empresas españolas. Universidad de Navarra. IESE Business School. OP 00/4. Artículo consultado el 18 de mayo de 2011 en:

http://www.iese.edu/research/pdfs/OP-00-04. pdf
Moneva, José Mariano (2006); iEs la Responsabilidad Social Corporativa rentable para la empresa? En Responsabilidad Social Corporativa, Barcelona, ACCID Contabilidad y Dirección, p.p. 55-76.

Moore, G. (2001): Corporate social and financial performance: an investigation in the UK supermarket industry. En Journal of Business Ethics. Vol. 34, No 3-4. p.p. 299 315, DOI: $10.1023 / \mathrm{A}: 1012537016969$

Orlitzky, M.,Schmidt, F. L.y Rynes, S.L. (2003); Corporate social and financial performance: A meta-analysis. En Organization Studies, Vol. 24, No 3. p.p. 403-441.

Paoli, María Celia (2002); Empresas e responsabilidade social: os enredamentos da cidadania no Brasil. En SANTOS, Boaventura de Sousa (Org.). Democratizar a democracia: os caminhos da democracia participava. Rio de Janeiro: Civilização Brasileira.

Periódico Globe and Mail, Canadá, Septiembre (2003); y cuestionario: The responsible Century, El siglo responsable, Burson-Marseller y The Prince of Wales Business Leaders Forum, www.bm.com.

Pinillos, Alberto Andreu (2005); La Responsabilidad Social Corporativa: un concepto por definir. En Revista de Economía Pública, Social y Cooperativa CIRIEC España. No 053. p.p. 125 - 135.

Ponencia para primer debate para el proyecto de ley No 326 de 2008 Cámara - 31 de 2007 Senado. Congreso de la República de Colombia.

Porter, Michael E. y Kramer, Mark R. (2006); Strategy and Society: the link between competitive advantage and Corporate Social Responsibility" Harvard Business Review,, Diciembre, 2006. p.p. 78-92. 
Porter, Michael E. y Kramer, Mark R. (2011); Creating Shared Value. How to reinvent capitalism - and unleash a wave of innovation and growth. Harvard Business Review, enerofebrero. p.p. 1-17.

Proyecto de Ley No. 31 de 2007, Congreso de la República de Colombia.

Rajas, R.G. y Zingales, L. (1998); Power in a theory of the firm. En Quarterly Journal of economics (2000): The governance of the new entreprise, Cambridge: University Press.

Ramírez, José Ernesto y Segura, Juan Carlos (2010); Las perspectivas de las organizaciones sociales para cambiar el actual panorama instrumental de la responsabilidad social empresarial en Colombia. En Revista Opera, Vol. 5, No 005. p.p. 97-119. ISSN 1657-8651

Raufflet, Emmanuel (2010); Responsabilidad corporativa y desarrollo sostenible: una perspectiva histórica y conceptual. En Cuadernos de Administración, No. 43. p.p. 23-42. ISSN 0120-4645.

Rodríguez, J.M. (2003); El gobierno de la empresa: un enfoque alternativo. Madrid: Akal, Economía Actual.

Rodríguez Calderón, Gisella (2010); La responsabilidad social empresarial: el reto que enfrentan las empresas a nivel mundial y nacional. Universidad Simón Bolívar. Artículo consultado el 18 de mayo de 2011 en:

http://www.unisimonbolivar.edu.co/ rdigital/desarrollogerencial/index.php/ desarrollogerencial/article/view/22/19

Rojas, Cristina. (2002); Corporate Philanthropy: A Reflection Base on the Colombian Experience. En Revista Harvard Review of Latin America, Spring. p.p. 27-29.
Sabino, Carlos (1992); El proceso de investigación. Caracas: Panapo.

Salamon, Lester M. (1999); Global Civil Society:Dimensions of the Nonprofit Sector. Baltimore: Johns Hopkins Center for Civil Society Studies.

Sanborn, Cinthya y Portocarrero, Felipe (Editores) (2003); De la caridad a la solidaridad: filantropía y voluntariado en el Perú. Lima: CIUP. p.p. 289-322.

Schmidheiny, S. (2004); La visión del empresario para el desarrollo sostenible a través de la responsabilidad social. En V Encuentro nacional de la empresa privada. Artículo consultado el 18 de mayo de 2011 en:

http://www.anep.org.sv/ENADE2004.

Simpson, W. G. y Kohers, T. (2002); The link between corporate social and financial performance: evidence from the banking industry. En Journal of Business Ethics, No. 35, p.p. 97-109.

Solarte Rodríguez, Roberto (s.f). La Responsabilidad Social de las Organizaciones. Situación y Perspectivas. En Papers Retos, Universidad Javeriana de Colombia. Articulo consultado el 16 de mayo de 2011 en:

http://www.javeriana.edu.co/Facultades/ Teologia/servicios_formacion_int_univ/ ecoteo1/pret-01b.pdf

Trujillo, María Andrea y Vélez Bedoya, Rodrigo (2006); Responsabilidad ambiental como estrategia para la perdurabilidad empresarial. En Univ. Empresa, Vol. 5, No 10. Universidad del Rosario. p.p. 291-308.

Vargas Sánchez Alfonso y Vaca Acosta, Rosa María (2005); Responsabilidad Social Corporativa y cooperativismo: Vínculos y 
Responsabilidad Social Corporativa (RSC) y Desarrollo Sostenible: una mirada desde la Declaración de Rio de 1992

potencialidades. En CIRIEC España, No 53.

p.p. 241-260. ISSN: 0213-8093.

Velásquez, M.G. (2000); Ética en los negocios. Conceptos y casos. México: Prentice HillPearson Educación.

Vélez, Ana María (2010); Una respuesta a la acción social, de la rentabilidad a la responsabilidad social empresarial. En Colombia Revista Ciencias Estrategicas, Vol. 23 p.p.11 - 25. Ed: Editorial Universidad Pontificia Bolivariana. ISSN: 1794-8347

Vidal, María del Mar Maira (2010); La Responsabilidad Social de las Empresas transnacionales con sede central en España, ¿Neopaternalismo industrial o globalización de derechos? En: Sociología del Trabajo, Nueva Época. No. 69. p.p. 95-118.

Visser, W. (2008); Corporate Social Responsibility in developing countries. En A. Crane, A. McWilliams, D. Matten, J. Moon $\&$ D. Siegel (eds.), The Oxford Handbook of Corporate Social Responsibility, Oxford: Oxford University Press, p.p. 473-479.

Vives, A., Corral, A. e Isasi, I. (2005); Responsabilidad Social de la Empresa en las PYMES de Latinoamérica BID-IKEI. Septiembre de 2005.

World Summit on Sustainable Development IISD, I. I. f. S. D., 2002. Summary of the World Summit on Sustainable Development: 26 August - 4 September 2002, Paper presented at the http://www.iisd.ca/linkages/ vol22/enb2251e.html; WSSD, 2002. Plan of Implementation, Johannesburg. 